\title{
Revisión sistemática Diagnóstico y tratamiento del trastorno por déficit de atención con hiperactividad
}

\author{
Agency for Health Care Policy and Research (actualmente Agency for Health Care Research and Quality). \\ Diagnosis of Attention-Deficit/Hyperactivity Disorder. Summary, Technical Review: Number 3, August 1999. \\ 'http://www.ahrq.gov/clinic/adhdsutr.htm
}

Treatment of Attention-Deficit/Hyperactivity Disorder.Summary, Evidence Report/Technology Assessment: Number 11.AHCPR Publication No. 99-E017,December 1999.

http://www.ahrq.gov/clinic/adhdsum.htm

\section{Objetivos}

Revisar la evidencia científica disponible sobre la prevalencia, la validez de varios métodos de evaluación diagnóstica y los tratamientos del trastorno por déficit de atención con hiperactividad (TDAH).

\section{Fuente y selección de los datos}

Los datos sobre prevalencia y diagnóstico de TDAH fueron tomados de 87 artículos y diez manuales de escalas de comportamiento seleccionados conforme a criterios de inclusión y exclusión entre más de 4000 citas. A través de un modelo multivariado* se valoró el efecto de la edad, el sexo, la herramienta diagnóstica y el contexto del estudio (comunitario o escolar). Solo se revisaron estudios publicados en lengua inglesa entre 1980 y 1997 que figuraran en MEDLINE, PsycINFO, listas de referencia de revisiones, artículos de investigación, así como en una guía provista por la American Academy of Child and Adolescent Psychiatry, en citas sugeridas por los miembros de la American Academy of Pediatricians y en una base de datos bibliográfica sobre estudios que usaron o evaluaron cuestionarios de comportamiento infantil (Child Behavior Checklists).

Respecto del tratamiento se limitó la búsqueda a estudios comparativos sobre eficacia y seguridad (ensayos controlados aleatorizados) en el corto y en el largo plazo sobre intervenciones farmacológicas y no farmacológicas para TDAH, así como para intervenciones combinadas e individuales. Se revisó MEDLINE (desde 1966), CINAHL (desde 1982), HEALTHStar (desde 1975), PsycINFO (desde 1984) y EMBASE (desde 1984). También la Cochrane Library y sitios de Internet de organizaciones que investigan este trastorno. Entre 2405 citas identificadas se seleccionaron 92 reportes, describiéndose 78 estudios diferentes. Se decidió que sería inapropiado un metaanálisis para resumir la evidencia ya que se encontró heterogeneidad clínica en los estudios (terapias evaluadas, poblaciones de pacientes, duración de tratamientos); inconsistencia en la medición de los resultados, baja calidad metodológica y deficitaria información sobre las herramientas de evaluación.

\section{Resultados principales herramientas diagnósticas}

sobre las

- La prevalencia de TDAH depende del sexo, de los criterios diagnósticos (DSM-III o DSM-III-R), del contexto de estudio (comunitario o escolar), siendo mucho mayor cuando no son considerados los criterios de deterioro de la actividad académica y del área del comportamiento. La edad no es un determinante significativo.

- Los niños con TDAH de la población general tienen una alta prevalencia de comorbilidad (trastorno de conducta: 35\%; ansiedad: 25\%; depresión: $20 \%$; trastornos de aprendizaje: $12 \%$ ). El $33 \%$ de los pacientes tiene más de una comorbilidad.

- La Escala de Conners revisada en 1997 contiene dos indicadores de buen poder de discriminación entre niños con TDAH y controles normales. Las Subescalas más efectivas para valorar la Hiperactividad son la DSM-III-R SNAP y la Conners Abreviated Teacher Questionnaire (CATQ, HI). La Subescala DSM-III-R SNAP demostró ser útil para el área de la Desatención y de la Impulsividad. Otras Escalas como el Cuestionario Barkley School Situations y el ACTeRS no mostraron utilidad.

- La plumbemia no se considera una herramienta diagnóstica útil. Existe asociación inversa entre la prevalencia de TDAH y los niveles de plomo en la poblaciones estudiadas.

- No hay relación entre disfunción tiroidea y TDAH, no avalándose el uso de pruebas de función tiroidea como rastreo de dicha condición.

- A pesar de los hallazgos en la TAC, la RMN y el electroencefalograma, estos estudios no son considerados útiles como método de rastreo para TDAH y tampoco lo serían las pruebas de Rendimiento Continuo que miden impulsividad, inatención y alerta.

\section{Resultados principales sobre el tratamiento}

- Los estimulantes (metilfenidato, dextroanfetamina y pemolina) son más efectivos que las intervenciones no farmacológicas; y la terapia combinada ofrece ventajas si se la compara con el empleo exclusivo de estimulantes o de intervenciones no farmacológicas.

- No se encontraron diferencias en la efectividad a corto plazo entre los tres fármacos estimulantes valorados (metilfenidato, dextroanfetamina y pemolina)

- Mientras se lo administra, el metilfenidato parece reducir los trastornos de conducta en los niños con TDAH. Los estudios muestran, independientemente del tratamiento utilizado, una tendencia general al mejoramiento con el transcurso del tiempo.

- Pocos estudios y de muestras pequeñas sugieren que la desipramina es más efectiva que el placebo.

- El litio no parece ser una alternativa efectiva en pacientes resistentes a los estimulantes.

- Los pocos estudios sobre tratamiento de TDAH en adultos sugieren que el metilfenidato y los antidepresivos pueden ser efectivos

- En general los efectos adversos a los estimulantes son leves y responden a los ajustes de la dosis. Sin embargo, hay pocos 
estudios a largo plazo, así como sobre el potencial abuso de los estimulantes.

\section{Conclusiones}

Es necesario realizar estudios a gran escala utilizando los mismos criterios diagnósticos para establecer la prevalencia del TDAH y de sus tres subtipos, así como nuevos trabajos en el área de neuroimágenes que continúen explorando las diferencias funcionales y estructurales en los cerebros de los niños con este diagnóstico.

Se necesitan estudios con diseños más rigurosos para establecer la efectividad y los efectos adversos de las intervenciones (ej. eficacia relativa de los psicoestimulantes y los antidepresivos tricíclicos respecto de clonidina, bupropión e inhibidores selectivos de la recaptación de serotonina) y para determinar la influencia de los factores comórbidos (ansiedad, depresión, etc.) en la respuesta al tratamiento. Teniendo en cuenta la complejidad de este trastorno, la frecuencia de comorbilidades y la variedad de recursos terapeúticos disponibles, será necesario un gran compromiso y acuerdo entre los diferentes grupos de investigación para poder obtener conclusiones válidas para la práctica clínica.

Fuente de financiamiento: Agency for Health Care Policy and Research.

\section{Comentario}

Utilizando sólo trabajos en lengua inglesa, estas revisiones han intentado proveer una sinopsis diagnóstica y terapéutica actualizada del TDAH con la meta de que los agentes y las organizaciones de salud cuenten con parámetros comunes para el diagnóstico y el tratamiento de esta condición.

EI TADH es uno de los trastornos psiquiátricos más comunes de la niñez. Destacamos su importancia por su alta prevalencia (3 a $5 \%$ durante la edad escolar según el DSM IV1), por sus efectos perjudiciales en la formación y en el nivel de vida de los niños afectados, especialmente si su tratamiento es inadecuado 0 insuficiente.

Se caracteriza por un núcleo sintomático persistente que inter fiere en las actividades sociales y académicas del niño: déficit de atención (distractibilidad), impulsividad en las emociones y los actos motores, baja tolerancia a la frustración y, sobre todo en varones, hiperquinesia (excesiva actividad motora carente de finalidad ejecutiva²).

EI TAHD se caracteriza por síntomas persistentes que interfiere en las actividades sociales y académicas del niño: déficit de atención, impulsividad en las emociones y los actos motores, baja tolerancia a la frustración e hiperquinesia.

Estos pacientes presentan mayor riesgo de sufrir accidentes debido a su hiperquinesia/impulsividad y a su falta de atención. $3,4,5,6$

Su detección en la población general infantil es importante ya que el tratamiento mejora el desempeño social y académico del niño. Por otro lado, la falta de un diagnóstico correcto y/o un tratamiento inapropiado conducen a situaciones de estrés en el niño y su familia y a perturbaciones en las relaciones interpersonales.

Quien padece el TDAH suele generar rechazo en las personas con las que interactúa, lo que puede derivar en problemas de conducta y agresividad secundaria. Si a esta situación se le suma el fracaso académico, se empeora la baja autoestima que padecen estos niños.

No sólo es un problema de Salud Pública por las consecuencias individuales descriptas previamente, sino que también es relevante su costo financiero desde la perspectiva social debido a que esta condición se asocia a un riesgo aumentado de desarrollar durante la adolescencia y la adultez conductas antisociales, abuso de sustancias, así como síntomas afectivos y de ansiedad. Por ejemplo, a través de un estudio epidemiológico retrospectivo de una población penal, Eyestone y col.8 destacan que uno de cada cuatro reclusos había sufrido TDAH y depresión durante su infancia.

EI TADH se asocia a un riesgo aumentado de desarrollar conductas antisociales, abuso de sustancias, síntomas afectivos y ansiedad.

El tratamiento de los niños con TAHD mejora su desempeño social y académico.

Para la detección del TDAH en la consulta general la mayoría de los autores coincide en la importancia de la historia clínica ya que no existen exámenes complementarios que sean patognomónicos de este trastorno1,3,4,5,6,7. En la anamnesis a padres y maestros se destacan preguntas dirigidas hacia:

1) La poca capacidad del niño para mantener la atención (¿realiza esfuerzos sostenidos en relación con una tarea, especialmente si es aburrida o rutinaria?, ¿pasa de una actividad que no ha completado a otra?, ¿falla en completar las tareas en ausencia de control externo?, ¿se dispersa fácilmente?, ¿suele perder sus útiles escolares?)

2) La dificultad para retrasar la gratificación (¿piensa antes de actuar?, ¿puede esperar su turno?, ¿interrumpe a otros en forma intempestiva?, ¿puede controlar sus impulsos?, ¿impresiona ser un niño atropellado, impaciente, "molesto"?, ¿cambia de humor fácilmente?);

3) La presencia de excesiva actividad irrelevante o inadecuada (impresiona inquieto o ansioso?, ¿cambia frecuentemente de posición, especialmente cuando no esta interesado en la actividad propuesta?, ¿parece que tuviera un motor?).

Estas manifestaciones deben presentarse en por lo menos dos ámbitos diferentes, suelen aparecer en espacios grupales o en actividades aburridas y/o rutinarias (ej. en la escuela, club o en las actividades diarias dentro del hogar); y tienden a disminuir cuando el niño interactúa con otra persona en una relación uno a uno o bien, frente a situaciones novedosas o que motiven su interés (ej. una evaluación breve en el consultorio). Los niños que respondan afirmativamente a estos tres grupos de preguntas deberían ser derivados a un especialista.

Además, es importante efectuar un genograma que abarque tres generaciones destacando si existen familiares directos con síntomas compatibles con esta entidad (las personas que tienen un hermano con TADH tienen un $30 \%$ más de probabilidad de desarrollarlo que la población general y un $50 \%$, si se trata de gemelos). También existe mayor prevalencia de trastornos del estado de ánimo, de ansiedad, del aprendizaje, trastorno antisocial y relacionado con sustancias en los familiares de estos niños $1,4,5,6,7$.

Para la detección del TDAH se destaca la importancia de la historia clínica. No existen exámenes complementarios patognomónicos. 
También se debe recordar que hay diversas entidades que pueden expresarse con hiperactividad y/o desatención: epilepsia, déficits sensoriales, hipertiroidismo, anemia ferropénica; problemas psiquiátricos (abuso de sustancias, trastornos afectivos, del aprendizaje, lenguaje, sueño y conducta, Gilles de la Tourette, etc.); retraso mental, efectos adversos de algunos fármacos como el fenobarbital y la teofilina; y trastornos reactivos a conflictos familiares y ambientales.

Aunque se hayan encontrado algunos hallazgos en los estudios de neuroimágenes, en el electroencefalograma y en el dosaje de distintos neurotransmisores y hormonas, todavía no se consideran válidos para confirmar el diagnóstico.

Podrían ser de cierta ayuda diagnóstica el antecedente de complicaciones obstétricas y perinatológicas (prematurez, hipoxia o infecciones perinatales, exposición a drogas durante el embarazo, desnutrición, infección del sistema nervioso central durante el período neonatal, etc.) así como algunos estigmas morfológicos (signos genéticos menores) como el hipertelorismo, el epicanto y los remolinos en el cuero cabelludo6,7. En la estrategia diagnóstica especializada debe tenerse en cuenta la evaluación psicopedagógica, neurológica, fonoaudiológica y psicomotriz. Por otro lado, existen versiones para padres y para maestros de cuestionarios y pruebas para la detección y el monitoreo de la respuesta al tratamiento de la desatención y la hiperactividad impulsividad (ej. Test de Conners).

Respecto del tratamiento, consideramos que deben ser indicados por profesionales con experiencia en su uso y mencionamos a los psicoestimulantes como primera elección farmacológica hasta que haya mas evidencia de buena calidad que avale el uso de otros fármacos.

Se estima que el 75 al $80 \%$ de los pacientes responde favorablemente a los psicoestimulantes, comprobándose una disminución de la distractibilidad e impulsividad, lo que conduce a mejorías académicas y de las relaciones interpersonales $4,5,6$. No existen criterios firmemente establecidos sobre cuánto tiempo es necesario mantener dicho tratamiento y acerca del manejo de los pacientes con comorbilidades (que no son pocos). Si bien se recomienda realizar un monitoreo estricto del crecimiento y un control de los efectos adversos más comunes, no hay suficiente información sobre los efectos adversos en el largo plazo4,5,6.

Los psicoestimulantes son el tratamiento farmacológico de primera elección. Se recomienda que sólo sean indicados por profesionales con experiencia en el tema.

Se recomienda realizar un enfoque multidisciplinario que contemple la formación de los padres (los grupos de orientación favorecen el intercambio de estrategias y ayudan a disminuir las frustraciones); y el entrenamiento de los docentes ya que el niño necesita un plan educativo personalizado que contemple la duración más breve de su período de atención, evitar que se siente lejos de la maestra y en lugares donde es fácil la distracción, etc. Además, estos pacientes pueden beneficiarse de técnicas de relajación enseñadas por un psicomotricista para encauzar la hiperquinesia y la impulsividad a través de la coordinación de su esquema corporal. Es importante la prevención de los accidentes dentro y fuera del hogar, la selección adecuada de la escuela (en lo posible, grupos no muy numerosos y docentes entrenados) y la valorización de los logros del niño para fortalecer su confianza

Se recomienda un abordaje interdisciplinario que contemple el entrenamiento de padres y docentes, las técnicas de relajación y la prevención de los accidentes.

Queda continuar investigando con unificación de los criterios diagnósticos, las diferencias estructurales y funcionales halladas en los estudios de neuroimagenes que permitan una mejor categorización de los pacientes; así como, con el objeto de mejorar las opciones terapeúticas con el menor perfil de efectos adversos, realizar ensayos clínicos que evalúen en el corto y largo plazo las nuevas moléculas psicofarmacológicas para el tratamiento del TDAH y los trastornos comórbidos.

\section{Dr. Sebastián Cukier}

[ Residente del Hospital Infanto-Juvenil Carolina Tobar Garcia ]

\section{Dra. Paula Chávez}

[ Médica especialista en Pediatria. Servicio de Atención Pediatrica Domiciliaria CEMIC. Concurrente del Hospital Infantojuvenil Carolina Tobar Garcia ]

\footnotetext{
Referencias

1. American Psychiatric Association. Manual diagnóstico y estadístico de los trastornos mentales. DSM-IV. Editorial Masson, París, 1996

Frances A, Pincus H. First M editores. DSM-IV. Manual diagnostico y estadistico de los trastornos mentales. Pichot P, coordinador de la edicion española. 1995. Masson.

2. Rosan, T. Agresividad e hiperquinesia en niños con TDAH. ALCMEON (Revista Argentina de Clínica Neuropsiquiátrica. Órgano de Difusión de la Fundación Argentina de Clínica Neuropsiquiátrica y de la Asociación Argentina de Psiquiatria). Año XI, vol 9, Nro 2, Oct. 2000. Disponible en internet. URL: www.alcmeon.com.ar

3. Michanie, C. Trastorno por deficit de atención con hiperactividad. ALCMEON No 17 (Revista Argentina de Clínica Neuropsiquiátrica. Órgano de Difusión de la Fundación Argentina de Clínica Neuropsiquiátrica y de la Asociación Argentina de Psiquiatría). Nro 2, Año 1996. Disponible en internet. URL: www.alcmeon.com.ar

4. Spencer, T et al. Pharmacotherapy of attention deficit hyperactivity disorder. Child and Adolescent Psychiatic Clinics of North America. vol 9, nro 1, january 2000.

5. Mamondez, C. Sindrome de Dispersión de la atención con o sin hiperactividad. En el Programa Nacional de Actualización Pediátrica PRONAP. Editado por la Secretaría de Educación Contínua de la sociedad Argentina de Pediatría. 1999. Módulo 4. Cap 2. Pag. 33 a 47.

Continua de la sociedad Argentina de Pediatría. 1999. Modulo 4. Cap 2. Pag. 33 a 47. Prog 6. Massaro, M. Trastornos por déficit de atención con hiperactividad. En el Programa Nacional de Actual
la sociedad Argentina de Pediatría. 1999. Módulo 4. Cap 2. Lecturas Complementarias. Pag. 54 a 57.

7. Biaggi, H. Trastorno por Deficit de la Atencion. Un resumen actualizado. ALCMEON 18. (Revista Argentina de Clínica Neuropsiquiátrica. Organo de Difusión de la Fundación Argentina de Clínica Neuropsiquiátrica y de la Asociación Argentina de Psiquiatría) Nro3-1996. Disponible en internet. URL: www.alcmeon com.ar

8. Eyestone, LL et al. An epidemiological study of Attention Deficit Hyperactivity Disorder and Major Depression in a male prison population. B. of the American Academy of Psychiatry and the Law 22(2):181-93,1994.
} 